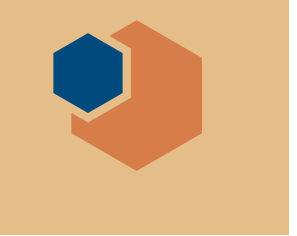

\section{Hard sell for metamaterials in the UK}

gov.uk/government/organisations/innovate-uk

A cademic researchers who want their new materials to become profitable technologies should not present their ideas as materials that can do anything and everything. They should focus on particular applications of their discoveries. That was the key message at a recent UK government-sponsored networking event "Connecting Local Industry to Metamaterials and Emerging Materials."

Organized by the Knowledge Transfer Network (KTN), the event at the University of Sussex brought together academic researchers and representatives of major companies such as Huawei, the defense giant BAE Systems, and the materials company Johnson Matthey, along with startups and investors. The meeting was a crash course for researchers and businesses on how to seek public funding to move ideas out of the laboratory into industry.

The KTN is a part of the government's innovation agency, Innovate UK (IUK), which, in turn, is a part of UK Research and Innovation (UKRI). Currently, UKRI has an annual budget of more than $£ 6$ billion, and exists to support businesses "to develop and realize the potential of new ideas including those from the UK's worldclass research base" (see the February 2018 issue of MRS Bulletin, doi:10.1557/ mrs.2018.15). The KTN's remit is to make connections between universities and industry. The Sussex event set out to explain how UKRI, along with government agencies such as the Defence and Security Accelerator, support innovation through a portfolio of business grants and loans. The event also addressed financing for early-career researchers to work in companies through IUK's Knowledge Transfer Partnerships.

Before money can flow to industry, there has to be business interest in the research. It is not always easy to attract business involvement with a subject like metamaterials, which may explain why there is a gap between how much UKRI

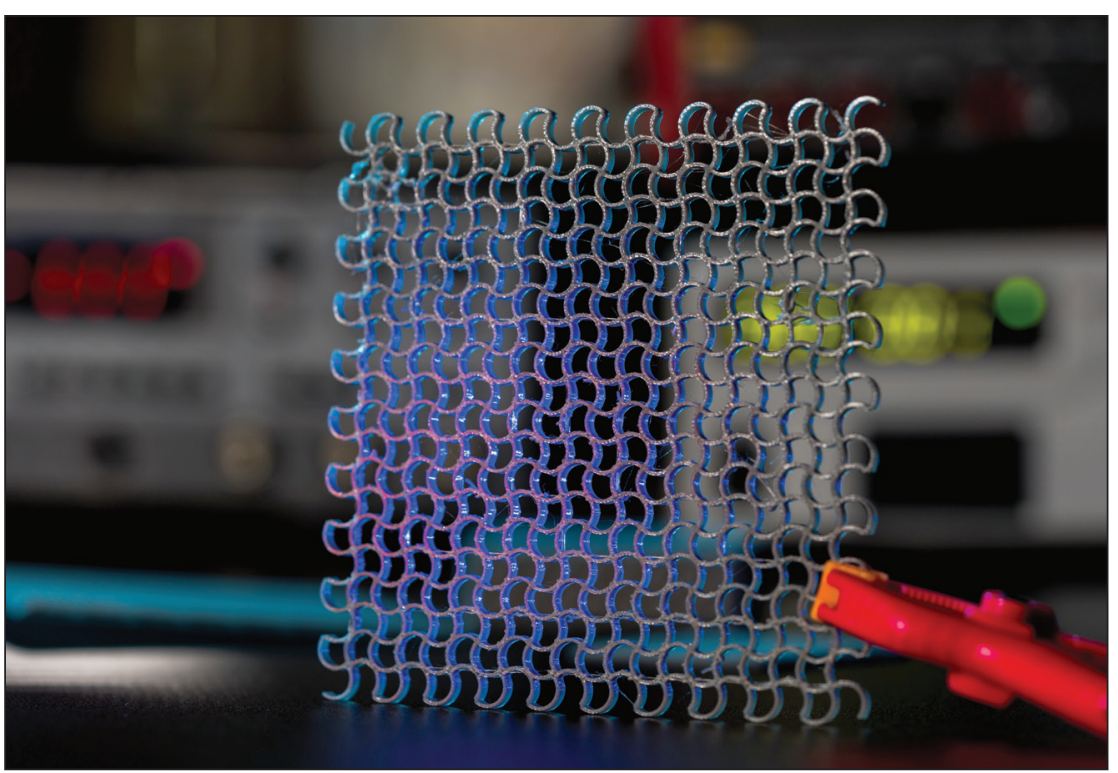

Acoustic metamaterial. Credit: Shutterstock.

has spent on academic research and IUK's investment in commercializing that work. Brian McCarthy, Knowledge Transfer Manager for Advanced Materials at the KTN, said at the meeting that the UK's research councils have invested around $£ 100$ million in metamaterials research, most of it from the Engineering and Physical Sciences Research Council (EPSRC). In contrast, when Lien Ngo, Innovation Lead for Advanced Materials with IUK, looked at the organization's record, she found just six projects that mentioned metamaterials amounting to "two and a bit million" pounds of funding.

Attempts to bridge this gap, and to drum up industrial interest in innovation, are a part of the UK government's Industrial Strategy, which aims to increase the amount of the gross domestic product (GDP) that goes into R\&D from $1.7 \%$ to $2.4 \%$ (see the February 2018 issue of MRS Bulletin, doi:10.1557/mrs.2018.15). The government has already committed an additional $£ 7$ billion of public funding up to 2022 as its contribution to reach the $2.4 \%$ target. In its delivery plan for 2019 , UKRI describes this as "the largest-ever increase in public funding of R\&D over a five-year period."

When it comes to signing up business support, McCarthy's definition of metamaterials showed how difficult it can be for potential users to grasp what they can do: "A metamaterial is a heterogeneous material that exhibits extraordinary or highly unusual properties arising from the combination of its structure and composition beyond those that a classical composite of the same constituent materials exhibits."

Ngo advised researchers that they should beware "the curse of the platform material." You may have a material that can modulate sound, it can modulate light, it can do everything. Focus on an application, she advised. "Pick something that a customer can recognize that they can use," she said.

Finding an application is just the beginning of the innovation process. Think also of the engineering, Ngo added. Many companies start in a university, they show that an idea will work and then think, as she put it, the rest is just engineering. "From my experience talking to lots of materials companies and working 
in materials spinouts, you have to put just as much time, effort, and money into that stage as you do into the actual materials discovery stage," she said.

Ngo urged researchers to think about potential applications of metamaterials by reference to the Industrial Strategy Challenge Fund, a part of the government's Industrial Strategy. This sets out to address challenges within four specific "pillars": aging society, artificial intelligence and data economy, future of mobility, and clean growth. "This is ... geared to 'how do we meet the big societal challenges?' "Ngo explained. For example, the challenges under "future of mobility" include self-driving vehicles, batteries for clean and flexible energy storage, "manufacturing and materials of the future," and "robots for a safer world."

This is where metamaterials researchers could look for suitable applications, Ngo said. As an example, she pointed to artificial intelligence and data economy and its challenge "Audience of the future" involving new immersive technologies as an obvious candidate. Think gaming, virtual reality, and other possible applications, was her message. "What do new immersive technologies look like?" she asked. Perhaps metamaterials could deliver useful acoustic applications.
Ngo advised that future challenges "won't say metamaterials. They probably won't even say materials." They will include such topics as "Manufacturing made smarter," "Commercializing quantum technologies," and "Driving the electric revolution." The idea is to let people bring their answers to these challenges, not to tell them what to do. Ngo said, "Use metamaterials to solve some of these challenges, that's how you need to think of it. Look at these challenges and think, 'How can the work I am doing help to solve some of those challenges?" "

Michael Kenward

\section{South Korea strengthens international ties for Industry 4.0}

Las

ast Spring, South Korea's Ministry of Science and ICT (MSIT) held separate joint committee meetings with Russia and Switzerland. The goal of the meetings was to expand cooperation on innovative technologies.

With Russia, South Korea agreed to focus on developing and commercializing technologies in artificial intelligence, high-functional UAVs for aerial and offshore missions, life science of developing anticancer immunotherapy, and quantum communications, identified as key drivers of the Fourth Industrial Revolution marked by accelerated technical progress.

Furthermore, the National Research Council of Science and Technology of South Korea and the Russian Academy of Sciences brought together about 40 experts in artificial intelligence, bioengineering, and advanced materials, and about 200 professionals from Russian universities and research institutes to share their research outcomes and to explore substantial cooperation methods between researchers. Both research institutes expect to continue this cooperation.
With Switzerland, MSIT and the National Research Foundation of Korea held a symposium at which 200 participants from firms, startup incubators, universities, and research institutes explored the future of the Fourth Industrial Revolution technologies, including artificial intelligence and the Internet of Things.

Members of both countries' industry, academia, and research institutes shared information on innovative technologies by giving presentations on smart transportation and safety, inclusive technologies for marginalized populations, technology-led solutions, and concepts of the smart city. They explored opportunities for research cooperation, investment, and market entry at a business matchmaking dinner.
US Department of Energy funds R\&D for fusion energy.gov

$\mathrm{T}_{\mathrm{s}}^{\mathrm{s}}$ he US Department of Energy (DOE) has recently announced USD \$14 million in funding for 10 university-led research projects in fusion energy. A major goal of the research is to develop methods of sustaining steady-state or continuous operation of fusion reactors, an essential step toward eventually making nuclear fusion a practical energy source.

"Fusion remains one of the world's most promising potential sources of energy," says Under Secretary for Science Paul M. Dabbar. "This researchaimed at achieving steady-state operation of fusion reactors-will be an important milestone on the road to sustainable energy from fusion," he adds.

Research will focus on high-priority challenges in magnetic confinement of plasma on the pathway toward eventual development of a contained, self-sustaining fusion reaction. The research will be performed on the DIII-D tokamak, the largest magnetically confined plasma facility in the United States. DIII-D is a DOE Office of Science user facility in San Diego, operated by General Atomics.

Projects range from developing new means of controlling plasma instabilities, to improving diagnostics, to advancing understanding of the material erosion that takes place inside fusion reactors.

More information about the 10 research projects is available on the DOE website at energy.gov. 